\title{
Analysis and Experimental Validation of the Tribological and Dynamic Characteristics of Journal Bearings
}

\author{
Xin Yang ${ }^{1 *}$, Genxiang Gu ${ }^{1}$,Sicong Sun ${ }^{2}$,Saili $\mathrm{Li}^{1}$,Jianming Zhou ${ }^{1}$ \\ 1 Shanghai Marine Diesel Engine Research Institute, Shanghai 201108, China \\ 2 School of Energy and Power Engineering, Wuhan University of Technology, Wuhan 430063, \\ China
}

\begin{abstract}
Sliding bearings are the main kinematic pair of a Stirling engine, and one of the main sources of friction power loss and excitation force. There is a coupling effect between the lubrication state and the excitation force. In this paper, the dynamic model of a Sapphire bearing fatigue test bed, which includes thermo-elasto-hydrodynamic (EHD) property, is established and the accuracy of the model is verified by the experimental data. Based on the model, the effects of the load, the oil supply temperature, the oil supply pressure, the bearing clearance on the tribological and dynamic characteristics are studied. With an increase in the load, the orbital path of the journal moves downward, the lubrication state changes from the full hydrodynamic lubrication to the boundary lubrication, the friction power loss increases, and the bearing excitation frequency increases. Due to the decrease of the oil viscosity, the hydraulic friction power loss decreases, but the friction power loss derived from the asperity contact increase, so does the total friction power loss. With the increase of the radial clearance, the shape of the orbital path and the minimum oil film thickness remain unchanged. The increase of the bearing clearance results in the collisions between the journal and bearing bush, thus the amplitude of the frequency multiplication component in the bearing load increases, and the asperity and the total friction power loss increase.
\end{abstract}

Keywords: journal bearing; bearing bush; elasto-hydrodynamic (EHD); multibody dynamics

\section{Introduction}

Journal bearings are widely used in Stirling engines, and they are the major kinematic pairs and the key source of power loss and vibration. Its tribological and dynamic characteristics have a significant impact on mechanical performance and reliability [1,2]. Therefore, it is of great importance to conduct an experiment and calculation study of the journal bearing characteristics on the tribology and the dynamics for a Stirling engine. There are three most important factors that influence the tribological and dynamic properties of the journal bearings: the dynamic properties of the moving parts, the tribological properties of the interface between the mating surfaces, and its experimental verification of the forces, which are very important but can hardly be measured directly in practice $[3,4]$.

In order to take the nonlinear effect of the oil film into account while maintaining the calculation precision, the degree of freedom (DOF) condensation method is widely used in a great deal of research. In this way, the solution accuracy and the speed can be satisfied at the same time [5-7]. The contact and the lubrication condition between the journal and the bearing bush affects the frictional coefficient and the oil film thickness. Patir, N. [8] applied the average flow model to

*Corresponding author: 13611633711@163.com 
take the various roughness configurations into account, which established the foundation of the EHD lubrication regime. To describe the friction force due to the asperity contact, Greenwood and Tripp [9] developed a theory based on the contact of two nominally flat, random rough surfaces. Bukovnik et al. [10] compared the different types of the simulation models of the journal bearings and the influence of the oil condition and came to the conclusion that the temperature has a significant impact on the oil viscosity. Wei et al. [11] discussed the flexibility of the parts while solving the EHD problem, and Zhang et al. [12] proposed an improved thermo-elastohydrodynamic (TEHD) lubrication, which included the flexibility of the bearing surfaces. The validation of the tribological and dynamic properties in the model is very essential [13-15]. Sun et al. [16] measured the orbit of the journal center of the crankshaft bearing under a number of conditions. Leclere et al. [15] studied the indirect measurement of the bearing loads. Mihara et al. [13] and Moreau et al. [14] developed an experimental measurement of the oil-film pressure and the oil-film thickness. Allmaier [17] and Priestner et al. [18] used Miba's journal bearing test rig to experimentally validate the simulation and get the refined simulation of the friction, including extensive oil and the wear profiles. Relying on a Sapphire bearing bush fatigue test bed, Ding et al. [19] and Qin et al. [20] studied the ultimate load of the journal bearings with the different materials and the surface treatment processes and made the assessments of the strength as well as the remaining useful life.

In the references above, the bearing lubrication and the dynamic characteristics are analyzed separately. For example, the unilateral influence of the tribology or the dynamics is only carried out by simulation or test method, and the influence of the boundary conditions on the tribology and the dynamics is not analyzed at the same time.

In this paper, a multibody dynamic model of the Sapphire bearing fatigue test bed (hereinafter called 'the test bed') is established by using the DOFs reduced method and the TEHD method, considering the influence of the contact surface morphology and the temperature on the lubrication characteristics. On this basis, the change in the law of the lubrication and the dynamic characteristics under the different working conditions is analyzed to provide guidance for optimizing the tribological and dynamic performance of the main bearings for the Stirling engine.

\section{Dynamics and Tribology Theory}

The multibody dynamic simulation model, which includes lubrication, involves dynamics and tribology. Due to the impact of the oil film, the pressure between the journal pin and the bearing bush can be transferred through two parts, which are the hydrodynamic pressure borne by the lubricating oil and the asperity pressure created by the asperity contact.

\subsection{Dynamic theory}

When establishing the shafting model, a simplified bearing is adopted to facilitate the calculation. The modal compression method is applied to the solution process in order to compress the journal and the shell in the same plane.

$$
\mathrm{M} \ddot{u}+C \dot{u}+\mathrm{K} u=F
$$

In the multibody dynamic analysis, the Guyan/Craig-Bampton method is used to reduce the freedom degrees of the crankshaft, and Equation (1) can be rewritten as follows [5,7]:

$$
\left[\begin{array}{ccc}
\mathrm{m}_{i i} & \cdots & \mathrm{m}_{i r} \\
\vdots & \ddots & \vdots \\
\mathrm{m}_{r i} & \cdots & \mathrm{m}_{r r}
\end{array}\right]\left[\begin{array}{c}
\ddot{u}_{i} \\
\vdots \\
\ddot{u}_{r}
\end{array}\right]+\left[\begin{array}{ccc}
c_{i i} & \cdots & c_{i r} \\
\vdots & \ddots & \vdots \\
c_{i i} & \cdots & c_{r r}
\end{array}\right]\left[\begin{array}{c}
\dot{u}_{i} \\
\vdots \\
\dot{u}_{r}
\end{array}\right]+\left[\begin{array}{ccc}
k_{i i} & \cdots & k_{i r} \\
\vdots & \ddots & \vdots \\
k_{i i} & \cdots & k_{r r}
\end{array}\right]\left[\begin{array}{c}
u_{i} \\
\vdots \\
u_{r}
\end{array}\right]=\left[\begin{array}{c}
f_{i} \\
\vdots \\
f_{r}
\end{array}\right]
$$

\subsection{Average Reynolds equation for rough surfaces}

During the operation of a Stirling engine, the crankshaft bears an alternating load with periodic changes in amplitude and direction. The stress and the deformation are produced on the surface of the bearing journal and the shell, which can change the oil film thickness. This difference in the thickness will result in the different contact forms between the journal bearing and the shell. The lubrication 
forms of the journal bearing are the boundary lubrication, the mixed lubrication, and the dry friction. The journal surface and the bearing shell mainly experience the dynamic pressure lubrication. When there is the mixed lubrication, the oil film thickness is only a few micrometers, and the influence of the asperity contact on the lubrication cannot be ignored. The equation used to solve for the lubrication is shown below [8]:

$$
-\frac{\partial}{\partial x}\left(\theta \phi_{x} \frac{\rho h^{3}}{12 \eta} \frac{\partial p}{\partial x}\right)-\frac{\partial}{\partial z}\left(\theta \phi_{z} \frac{\rho h^{3}}{12 \eta} \frac{\partial p}{\partial z}\right)+\frac{\partial}{\partial x}\left(\theta \rho\left(\bar{h}+\sigma \phi_{s}\right) \frac{U}{2}\right)+\frac{\partial(\theta \rho \bar{h})}{\partial t}=0
$$

\subsection{The Greenwood/Tripp contact model}

When the oil film thickness is very small, the asperity summits collide and produce a large friction force. Greenwood / Tripp [9] proposed an elastic contact theory, considering the nominal flat of the rough surface and the contact pressure force due to the asperity contact:

$$
\begin{gathered}
p_{\mathrm{a}}(h)=K E^{\prime} F_{\frac{5}{2}}\left(H_{s}\right) \\
H_{s}=\frac{h-\bar{\delta}_{s}}{\sigma} \\
E^{\prime}=\frac{1}{\frac{1-\varepsilon_{1}^{2}}{E_{1}}+\frac{1-\varepsilon_{2}^{2}}{E_{2}}} \\
F_{\frac{5}{2}}=\left\{\begin{array}{cc}
4.4086 \cdot 10^{-5}\left(4-H_{s}\right)^{6.804} & H_{s}<4 \\
0 & H_{s} \geq 4
\end{array}\right.
\end{gathered}
$$

\subsection{Viscosity-temperature characteristic equation of lubricating oil}

The viscosity-temperature-pressure characteristics of the lubricating oil should be comprehensively considered in the calculation process because the oil dynamic viscosity changes according to the temperature and the pressure. Based on the Vogel and Barus models [21], the viscosity relation of the lubricating oil was obtained as follows:

$$
\eta=A e^{\frac{B}{T-C}+\alpha p}
$$

\section{Multibody Dynamic Model of a Test Bed}

\subsection{Multibody dynamic model}

The structure of a test bed mainly consists of the base plate, the driving motor and the driving shaft system, the torquemeter and the test unit, as well as the hydraulic and the oil supply system. Its structure is shown in Fig.1. The tested unit consists of two supporting bearings, one connecting rod, and the tested shaft. The driving motor provides the speed boundary conditions for the tested shaft through the transmission shaft, and the hydraulic actuator applies the load to the tested bearing through the connecting rod. The lubrication system provides the stable oil supply boundary conditions for the bearings in the system, and the torquemeter measures the torque produced by the friction of the tested part. 


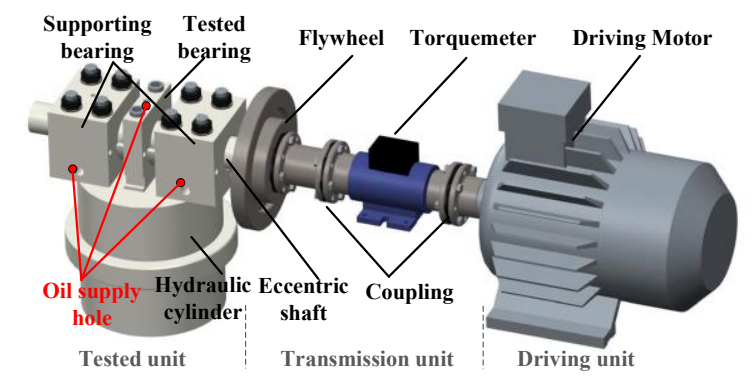

Fig. 1 Schematic drawing of test bed

Because there is no macro motion of other parts, except the tested unit, only the fixed and moving parts of the tested unit are considered as the flexible bodies in the modeling. The hydraulic load is inputted as the external load, and the movement of the driving unit is considered by the constant speed of the right node. The test bed comprises the four journal bearings installed in two bearing walls and one tested bearing installed in the connecting rod. The established dynamic model is shown in Fig. 2(a). The model includes two bearing walls, the measured shaft, and the connecting rod. There are two EHD kinematic pairs between the shaft and each bearing wall and one EHD kinematic pair with the connecting rod, as shown Fig. 2(b). The hydraulic load curves under the rated working conditions is shown in Fig. 2(c).

(a)

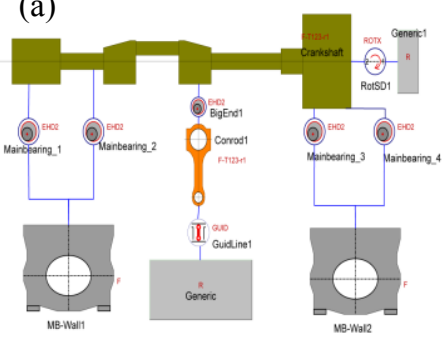

(b)

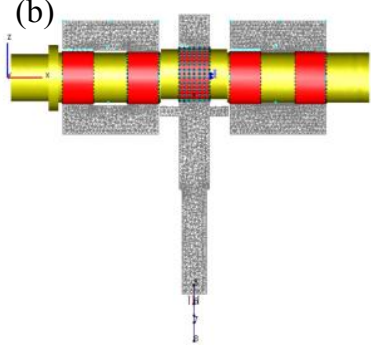

(c)

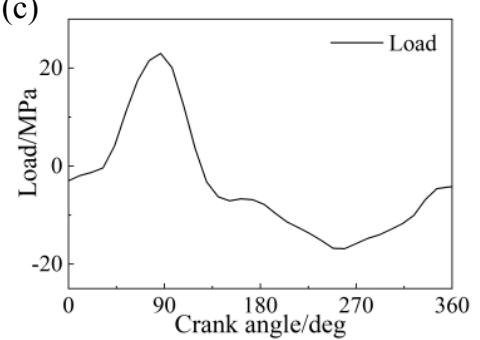

Fig. 2 Multibody dynamic model for test part of the test bed: (a) 2D model, (b) 3D model, (c) Pattern of loading in the test bed

\subsection{Multibody dynamic simulation model construction}

The measured component is the most important part in the test bed. The accuracy of the mass and the stiffness matrixes of each component directly affects the accuracy of the calculation results. In order to improve the calculation efficiency, the condensed body is established as shown in Table 1. In order to ensure that the dynamic characteristics of the condensed body are consistent with the finite element model and reduce calculation error, the modal calculation of the finite element model and the reduced model is carried out respectively. The comparison of the modal frequencies of each order is shown in Table 2 and Table 3.

Table 1. FE and condensed model of moving parts

\begin{tabular}{ccccc}
\hline \multicolumn{1}{c}{ Part } & Finite element model & Condensed model \\
\hline Shaft & & & \\
Bearing Wall & & & \\
Connecting rod & & & \\
\hline
\end{tabular}


Table 2. Modal frequency comparison between FE and condensed mode of shaft

\begin{tabular}{|c|c|c|c|c|}
\hline \multirow{2}{*}{$\begin{array}{l}\text { Modal } \\
\text { order }\end{array}$} & \multicolumn{2}{|c|}{ Frequency $/ \mathbf{H z}$} & \multirow{2}{*}{$\begin{array}{c}\text { Error } \\
/(\%)\end{array}$} & \multirow{2}{*}{ Modal shape } \\
\hline & FE model & Condensed model & & \\
\hline 1 & 1708.5 & 1710.5 & 0.117062 & Bending mode in $\mathrm{YZ}$ plane \\
\hline 2 & 1718.7 & 1721.7 & 0.174551 & Bending mode in $\mathrm{XZ}$ plane \\
\hline 3 & 4021.6 & 4020.4 & -0.02984 & Torsion mode \\
\hline 4 & 4332.6 & 4344.5 & 0.274662 & Bending mode in $\mathrm{YZ}$ plane \\
\hline 5 & 4360.8 & 4362.9 & 0.048156 & Bending mode in $\mathrm{XZ}$ plane \\
\hline Modal & \multicolumn{2}{|c|}{ Frequency/Hz } & Error & \\
\hline order & FE model & Condensed model & $/(\%)$ & Modal shape \\
\hline 1 & 963.5 & 963.23 & -0.02802 & Bending mode in $\mathrm{XZ}$ plane \\
\hline 2 & 1213.6 & 1212.4 & -0.09888 & Torsion mode \\
\hline 3 & 1558.6 & 1554.6 & -0.25664 & Bending mode in $\mathrm{YZ}$ plane \\
\hline 4 & 1816.4 & 1814.4 & -0.11011 & Local modes in small end \\
\hline 5 & 2644.1 & 2645.1 & 0.03782 & Torsion mode \\
\hline
\end{tabular}

The results show that the errors of the modal frequencies of the shaft and connecting rod condensation body with the finite element model are less than $1 \%$, which shows that the dynamic characteristics of the reduced model and the finite element model are consistent.

\subsection{Properties of kinematic pair}

The actual surface profile can be considered as the superposition of the contour centerline and the roughness profile, as shown in Fig. 3 and Fig. 4 respectively. The contour center line is determined by the nominal geometric size, the machining error, the installation deformation, and the thermal deformation. The thermal profile deformation of the journal bearing bush is calculated based on the measured experimental data.

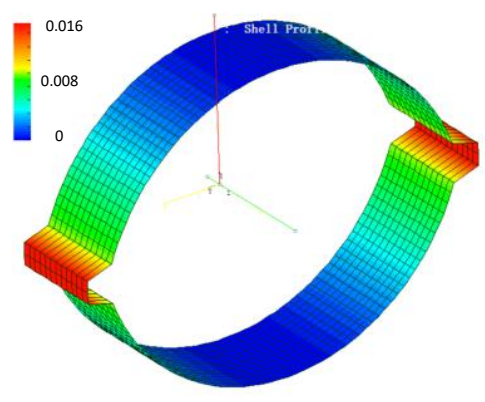

Fig. 3 Thermal profiles of bearing bush

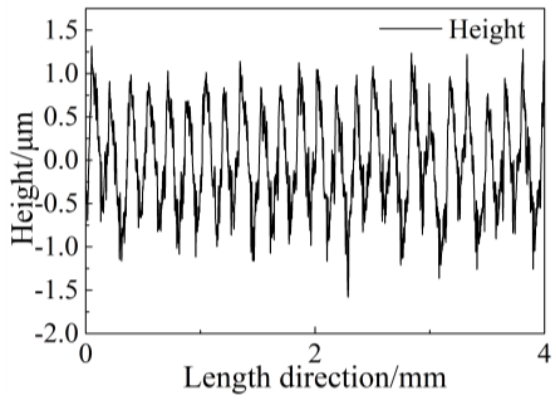

Fig. 4 Roughness profile of bearing bush Table 4. Statistical parameters of roughness profile

\begin{tabular}{cccc}
\hline Surface & $\boldsymbol{R a}(\mu \mathrm{m})$ & $\boldsymbol{R} \boldsymbol{q}(\mu \mathrm{m})$ & $\boldsymbol{R z}(\mu \mathrm{m})$ \\
\hline Journal & 0.474 & 0.558 & 2.525 \\
Bearing Bush in Support Seat & 0.492 & 0.580 & 2.601 \\
Bearing Bush in Connecting rod & 0.891 & 1.186 & 4.719 \\
\hline
\end{tabular}


The surface roughness measured by the Mitutoyo roughness meter is shown in Table 4 . According to Patir $\mathrm{N}$ and Greenwood [8], the flow coefficient and contact factor are obtained as shown in Fig. 5 and Fig.6.

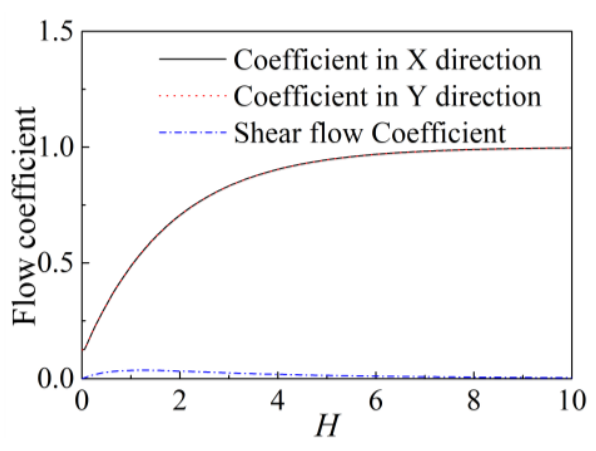

Fig. 5 Flow coefficient of bearing

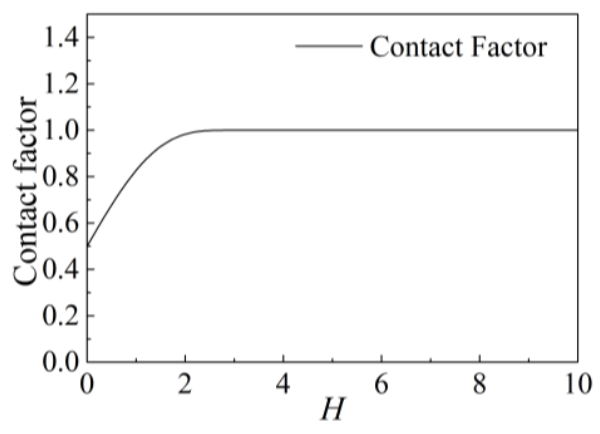

Fig. 6 Contact factor of bearing bush

\section{Experiment}

In order to ensure the correctness of the calculation model, it is necessary to carry out a verification test on the test bed. A schematic drawing of the test bed is shown in Fig. 1. The physical diagram of the test parts is shown in Fig. 7. The main measurement parameters of the test bed include the orbital path of the tested shaft, the bearing bush temperature, the friction power loss, the vibration on the test bed, the oil supply pressure, and the top dead center (TDC) signal. The main test parameters are shown in Table 5. The experimental flow is shown as in Fig. 8.

(a)

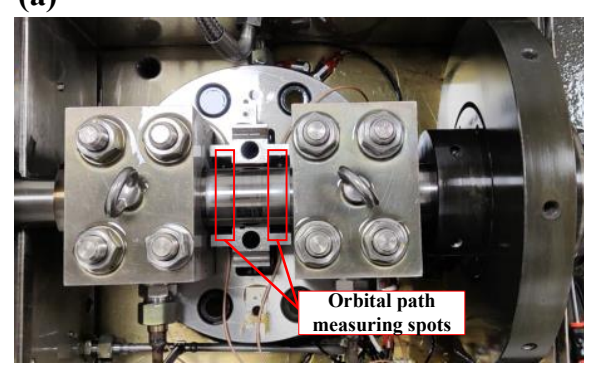

(b)

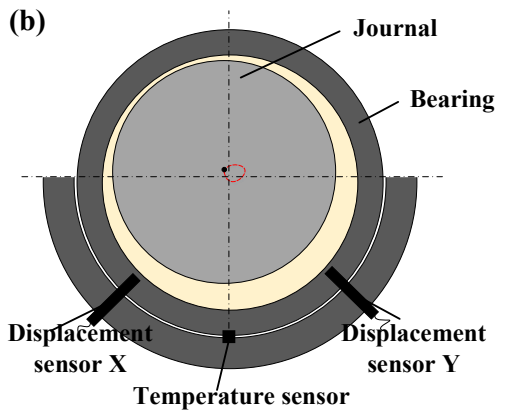

Fig. 7 Measured points in the test bed: (a) all sensor-installed positions, (b) Displacement and temperature sensor installation position

Table 5. The measured sensor signals

\begin{tabular}{ccc}
\hline Sensor & Signal & Description \\
\hline Pressure sensor & Load & The cyclic load applied to bearings \\
Displacement sensor & Displacement & Orbital path \\
Accelerometer & Vibration & Bench vibration \\
Temperature sensor & Temperature & Bearing bush temperature \\
Supply pressure sensor & Pressure & Lubrication supply pressure \\
\hline
\end{tabular}




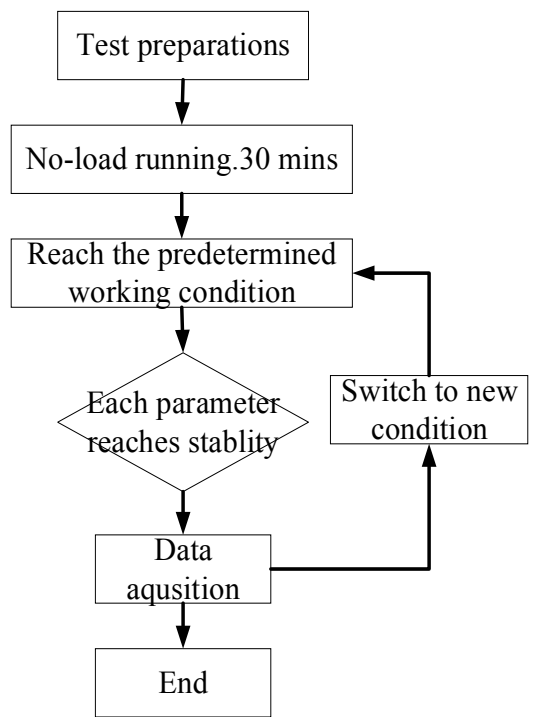

Fig. 8 The testing flow chart of the test

\section{Result and Discussion}

\subsection{Model verification}

It was found that the variation trend of the experimental friction power loss was consistent with that calculated under the different working conditions, as shown in Fig.9 (a). Meanwhile, the experimental orbital path has shape similarity with that of the calculated orbital path, as shown in Fig.9 (b), which indicates that the model is correct in dynamics property.

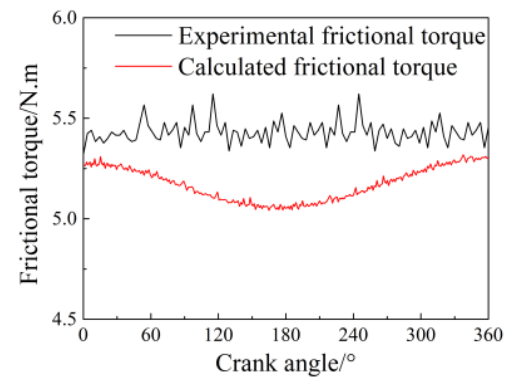

(a)

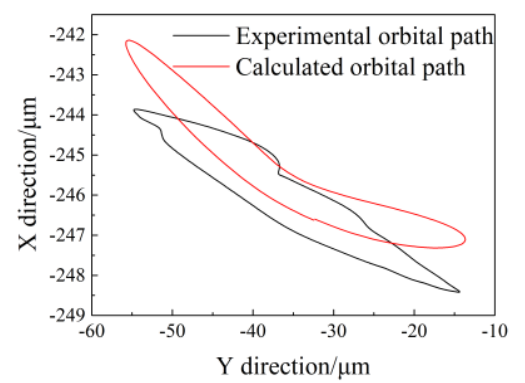

(b)

Fig. 9 Comparison of experimental and calculation results: (a) Frictional torques experimented and calculated, (b) Orbital paths experimented and calculate

\subsection{Tribological and dynamic characteristics in different boundary conditions}

\subsubsection{Tribological properties under the different working conditions}

Tribological property is a very important characteristics of journal bearings. The bearing characteristics are usually evaluated from the orbital path, the oil film thickness, the friction power loss, the hydraulic and the asperity film pressure distribution. For the lubrication performance of the journal bearings, the main factors include the amplitude of the external load, the lubricating oil temperature, the oil supply pressure and the bearing clearance. The lubrication characteristics of the bearings in different conditions will be discussed in this part.

Fig. 10 (a) shows the variation trend of the orbit paths with a variation in the loads. It can be found that when the load is very small, the orbit path is similar with the ideal circle, and the shaft will move around the surface of the bearing bush. When the load increases, the orbit of the shaft center gradually moves downward and concentrates at the lower bearing bush, and the shape of the orbital path becomes more and more irregular. This indicates that the lower bearing gradually enters 
the boundary lubrication regime under the excitation force after increasing the load, which is consistent with the wear phenomenon of the lower bearing, as shown Fig. 11. Fig. 10 (b) shows the change of the orbit paths with the oil supply temperature. It can be found that when the load is constant, with an increase of the oil supply temperature, the shape of the orbital path is unchanged, and gradually moves downward under the effect of the loads. This reflects a decrease in the oil film supporting capacity due to the decrease of the oil viscosity for the temperature increase. Fig. 10 (c) shows the variation trend of the orbit paths with the oil supply pressure. It can be observed that with an increase of the oil supply pressure, the orbital path does not change, which suggests that under a certain load when the oil supply is sufficient, increasing the oil supply pressure has little effect on improving the lubrication condition. Fig. 10 (d) shows the variation trend of the orbital paths with the different bearing clearances. The shape of the orbital path and the minimum oil film thickness remain unchanged with the increase in the clearance.

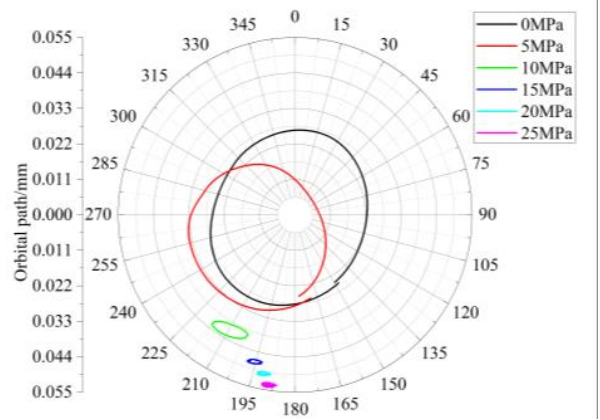

(a)

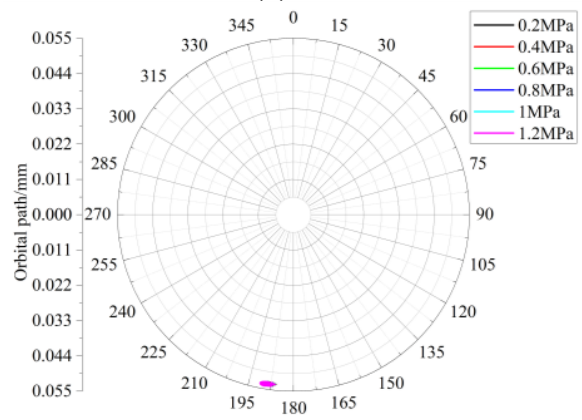

(c)

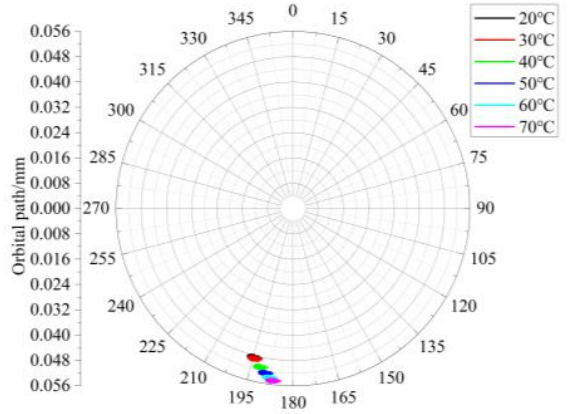

(b)

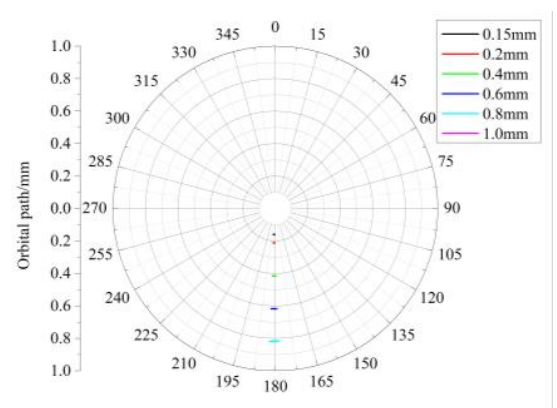

(d)

Fig. 10 Orbital path in different conditions: (a) Orbital path within different loads, (b) Orbital path within different oil supply temperatures, (c) Orbital path within different oil supply pressure, (d) Orbital path within different radial clearances

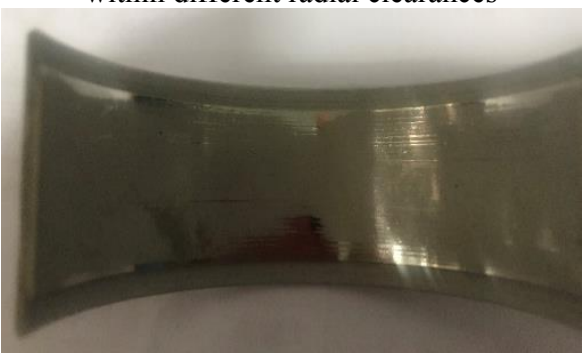

Fig. 11 Wear in bearing bush

The variation trend curves of the minimum oil film thickness with the different loads are shown in Fig. 12 (a). Corresponding to Fig. 10 (a), it can be observed that when the load is small, the minimum oil film thickness is larger. As the load increases, the minimum oil film thickness gradually decreases. When the load is $5 \mathrm{MPa}$, the reciprocating inertia force is close to the external load at $270^{\circ}$ crank angle (CA), thus the oil film pressure is under uniform distribution and result in a big oil film thickness. The variation trend of the minimum oil film thickness with a CA under the different oil supply temperatures are shown in Fig. 12 (b). It is not surprising to find that when the oil supply temperature increases, the minimum oil film thickness decreases until it reaches a stable state, which indicates that the increase in the oil supply temperature makes the lubricating 
oil viscosity and the oil film bearing capacity decrease. Fig. 12 (c) shows the minimum film thickness curve of the different oil supply pressures. The minimum oil film thickness changes very little within this range of the oil supply pressure, so does that of the Fig. 12 (d). In addition, the minimum oil film thickness gradually becomes a straight line in a cycle because the orbital path is stable at a certain position.

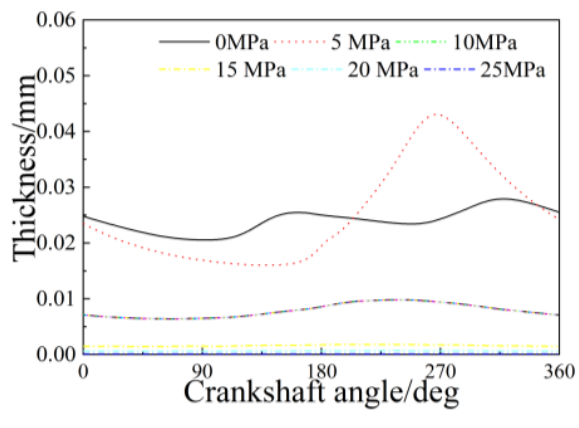

(a)

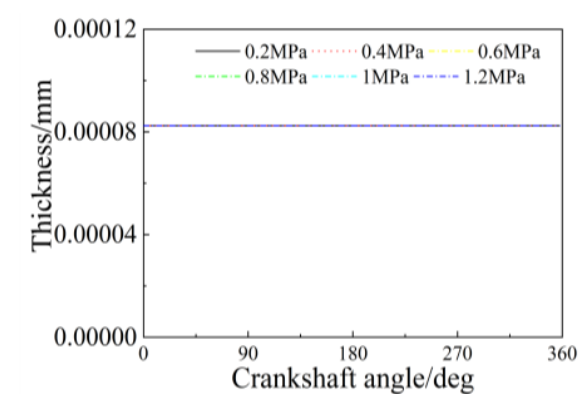

(c)

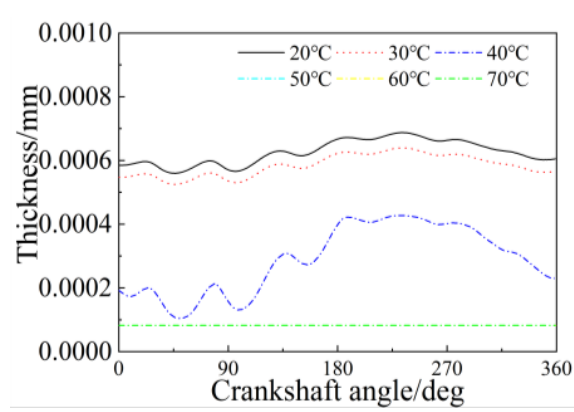

(b)

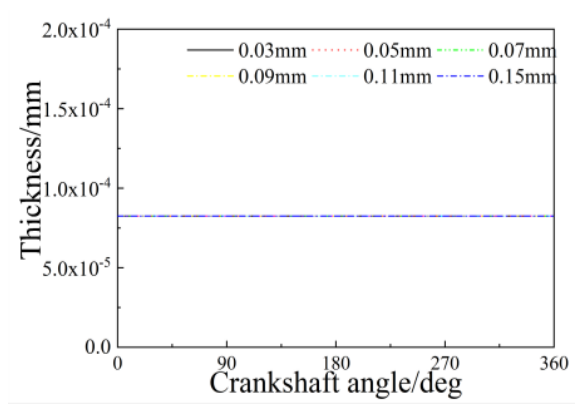

(d)

Fig. 12. Minimum oil thickness in different conditions: (a) Minimum oil thickness within different loads, (b) Minimum oil thickness within different temperatures, (c) Minimum oil thickness within different supply pressures, (d) Minimum oil thickness within different radial clearances

The variation trend of the friction power loss under different loads is shown in Fig. 13 (a). With an increase in the load, the friction power loss increases. The friction power loss also increases with an increase in the oil supply temperature as it is shown in Fig. 13 (b), while the hydraulic friction power loss decreases with an increase in the oil supply temperature as it is shown in Fig.13 (c). Fig.13 (d) shows the oil supply in certain range can hardly influence the friction power loss for the big difference in pressure magnitude orders between the supply oil and that of the oil film. From Fig.13 (e), it is easy to find that the friction power loss goes higher with the increase of the clearance, while the friction power loss derived from asperity contact goes downward and then rises with the increase of clearance as it is shown in Fig.13 (f). It is should not be ignored that the friction power loss caused by asperity contact increases disproportionately, as it changes more and more sharply when the clearance gets rather big. This indicates that the small clearance involves stable lubrication condition but it also could result in the more frequent asperity contact when the clearance is too small, while the big clearance means the increase of minimum oil film thickness but it could move in the opposite way when the clearance is too big with the more frequent collision between the journal and the bearing bush. 


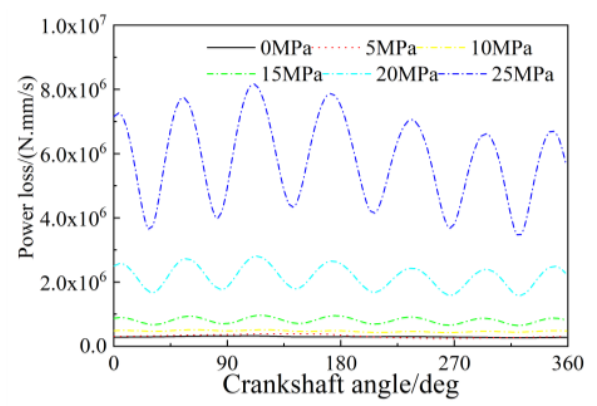

(a)

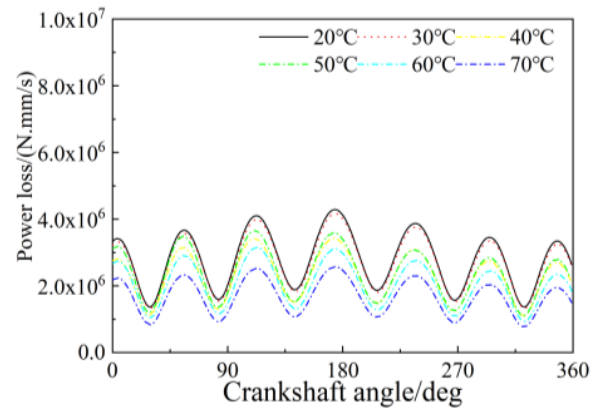

(c)

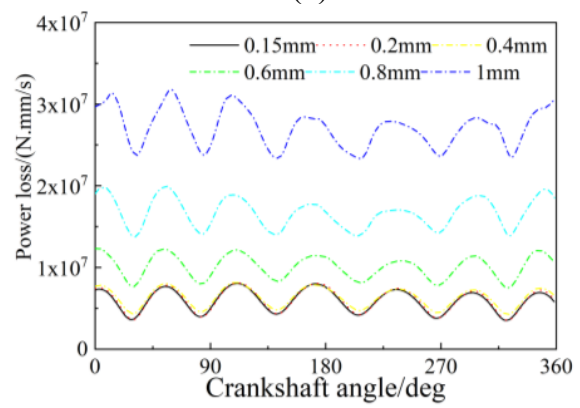

(e)

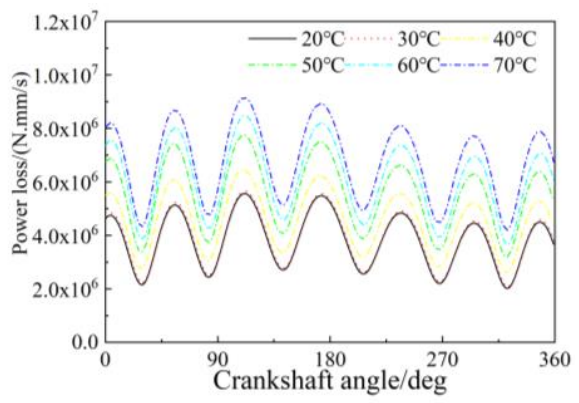

(b)

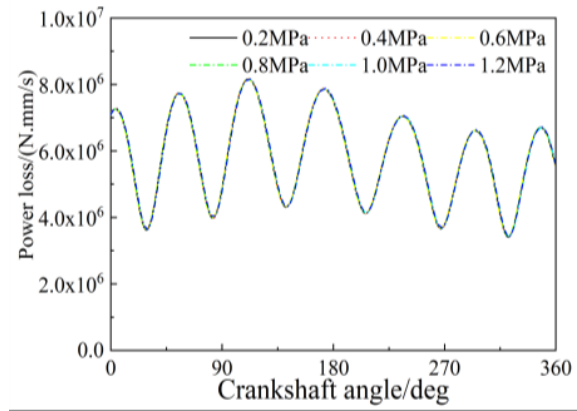

(d)

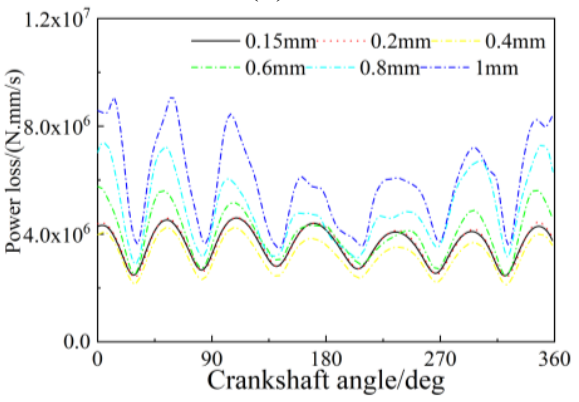

(f)

Fig. 13. Fictional power loss in different conditions: (a) Power loss within different loads, (b) Power loss within different temperatures, (c) Power loss within different supply pressures, (d) Hydrodynamic power loss within different temperatures, (e) Power loss within different radial clearances, (f) Asperity power loss within different radial clearances

Fig. 14 (a) and Fig. 14(b) respectively show the total and asperity contact oil film pressure distribution at the thinnest oil film position of the different orbital path in different external loads. Based on the two pictures, when the load exceeds $15 \mathrm{MPa}$, the orbital path mainly gets more and more close to the lower bearing bush, which results in the soaring of the total oil film pressure and the asperity contact pressure. Because the bending of the journal caused by the load, the asperity contact pressure mainly concentrates on the edge of the bearing bush. In this way, the tested bearing transfers into the mixed lubrication from the hydrodynamic lubrication.

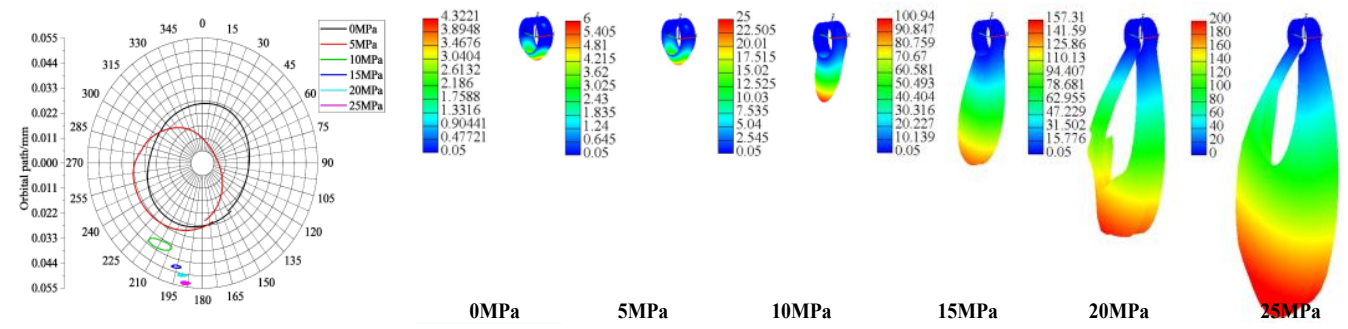

(a) 


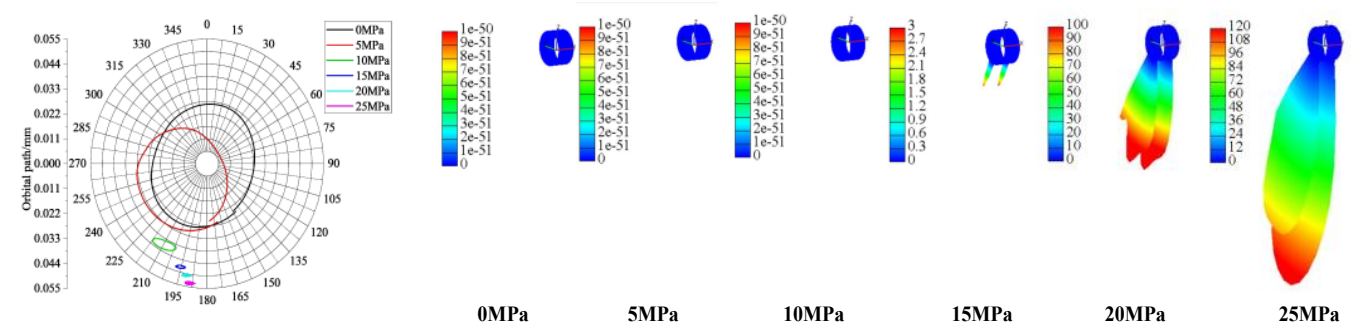

(b)

Fig. 14. The testing flow chart of the fatigue test: (a) Total pressure distribution varied with different load, (b) Asperity contact pressure distribution varied with different loads

\subsubsection{Dynamic properties under different operating conditions}

The coupling effects between tribological and dynamic properties should not be ignored. In this part, the influences of the different factors including the tribological aspects on the excitation forces are studied both in time domain and in frequency domain.

Fig. 15 shows the force in the big end of the connecting rod under the different load conditions. With the increase of the load, the amplitude of the force both in time domain and in frequency domain rises, as Fig 15 (a) and Fig 15 (b) shown respectively. Comparing Fig 10 (a) and Fig 15 (b), it can be found that when the load increases, the amplitude of the frequency multiplication in the excitation force gradually increases, which mainly derives from the change of the orbital paths.

Although the temperature has rather great influence on the lubrication condition as it is shown in Fig.13 (b), it doesn't influence the dynamic properties as Fig.15 (c) and Fig.15 (d) shown. By comparing Fig.15 (e) and Fig.15 (f), it is obvious that the oil supply pressure has little influence on the bearing dynamic property.

Similar to the influence the clearance has on lubrication, the clearance also influences the dynamic performance of the bearing as Fig.15 (g) and Fig.15 (h) shown. Although, the bearing load does not increase significantly in time domain, the amplitude of the frequency multiplication component in the excitation force increases in frequency domain. As Fig.13 (f) and Fig. 15 (b) described, the change in the clearance will result in the transformation of the orbital paths, and the frequent collision between the journal and the bearing bush creates the rise of the amplitude in frequency multiplication component.

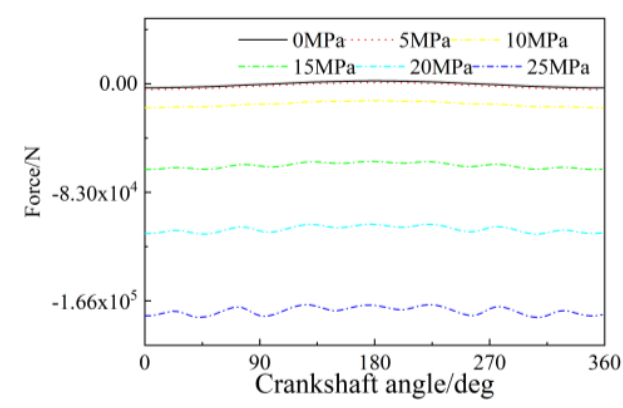

(a)

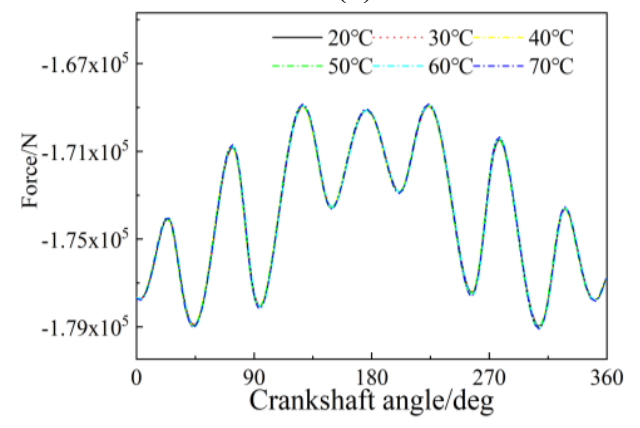

(c)

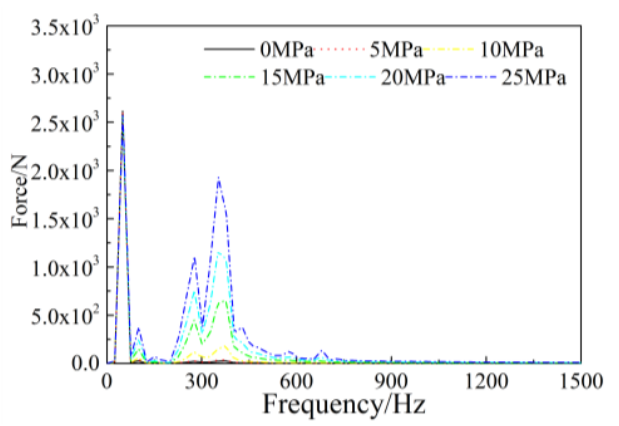

(b)

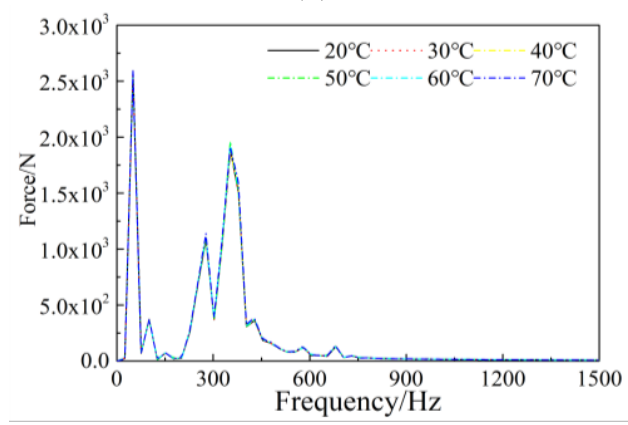

(d) 


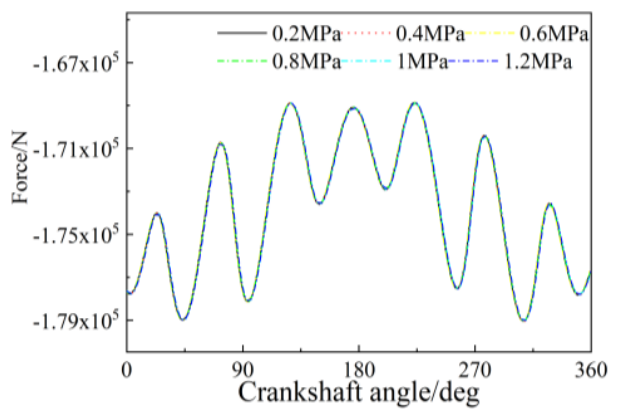

(e)

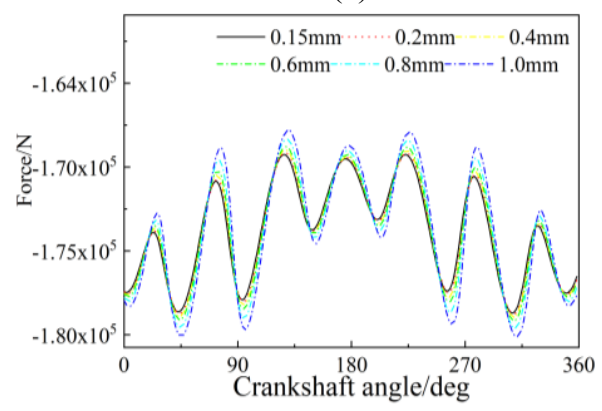

(g)

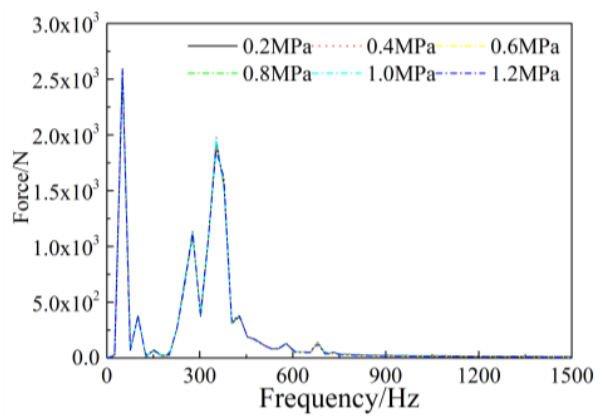

(f)

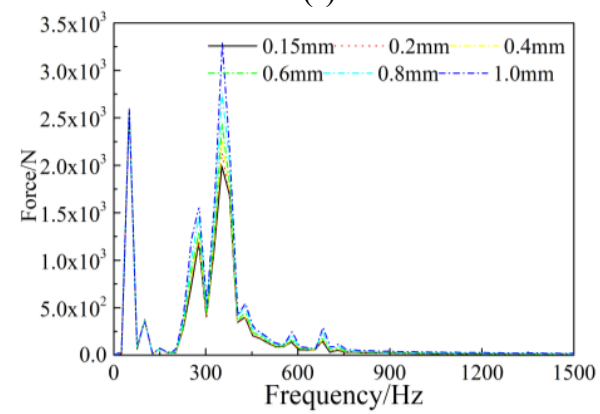

(h)

Fig. 15. The excitation force of bearing: (a) Load of bearing in time domain varied in different loads, (b) Load of bearing in frequency domain varied in different loads, (c) Load of bearing in time domain varied in different oil temperatures, (d) Load of bearing in frequency domain varied in different oil temperatures, (e)

Load of bearing in time domain varied in different supply pressures, (f) Load of bearing in frequency domain varied in different supply pressures, $(\mathrm{g})$ Load of bearing in time domain varied in different bearing radial clearances, (h) Load of bearing in frequency domain varied in different bearing radial clearances

\section{Conclusions}

A multibody dynamic model of TEHD for a test bed is established and verified by test results in this paper. The correctness of the calculation model and the research method was verified from the two aspects of the tribology and the dynamics.

The effects of the different working conditions on the tribological properties were compared. With an increase of the load, the shape of the orbital paths gradually changes from a circle to the irregular and move downward to the lower bearing bush. The minimum oil film thickness decreases drastically, and the friction power loss increases.

With an increase in the oil supply temperature, the oil viscosity decreases, thus the oil film supporting capacity weakens, the shape of the orbital paths remains unchanged but gradually moves downward and the minimum oil film thickness decreases. The decrease of the viscosity involves the decreases of the hydraulic friction, while the asperity contact power near the minimum oil film thickness rises as the oil film supporting capacity decrease. However, the influence of the oil supply pressure on the orbital path and the friction power loss is not obvious due to the significant difference in the order of the magnitude.

With the decrease of the clearance, the orbital path moves downwards, while the minimum oil film thickness remain the same, and the friction power loss caused by the asperity contact increases disproportionately, as it changes more and more sharply when the clearance gets rather big. The small clearance involves the stable lubrication condition but it also could result in the more frequent asperity contact when the clearance is too small, while the big clearance means the increase of the minimum oil film thickness but it could move in the opposite way when the clearance is too big with the more frequent collision between the journal and the bearing bush.

The effects of the different working conditions on the dynamic characteristics were compared. The oil temperature and supply pressure have little impact on force.

With the increase in the load, the orbital path gradually deviates from the circle, the frequency multiplication in the excitation force gradually increases. The change in clearance will result in the 
transformation of the orbital path, and the frequent collision between the journal and the bearing bush in the extreme condition creates the rise of the amplitude in the frequency multiplication component while creating the more friction power loss.

\section{References}

1.Taylor, C.M., Automobile engine tribology - design considerations for efficiency and durability. Wear 1998, 221, 1 .

2.Tung, S.C.; McMillan, M.L., Automotive tribology overview of current advances and challenges for the future. Tribol Int 2004, 37, 517.

3.Wang, D.; Keith, T.G.; Yang, Q.M.; Vaidyanathan, K., Lubrication analysis of a connecting-rod bearing in a high-speed engine. Part i: Rod and bearing deformation. Tribol T 2004, 47, 280-289.

4.Wang, D.; Keith, T.G.; Yang, Q.M.; Vaidyanathan, K., Lubrication analysis of a connecting-rod bearing in a high-speed engine. Part ii: Lubrication performance evaluation for non-circular bearings. Tribol T 2004, 47, 290-298.

5.JR., R.R.C.; BAMPTON, M.C.C., Coupling of substructures for dynamic analyses. AIAA Journal 1968, 6, 1313-1319.

6.Brüls, O.; Duysinx, P.; Golinval, J.-C., The global modal parameterization for non-linear modelorder reduction in flexible multibody dynamics. Int J Numer Meth Eng 2007, 69, 948-977.

7.Wu, L.; Tiso, P., Nonlinear model order reduction for flexible multibody dynamics: A modal derivatives approach. Multibody Syst Dyn 2016, 36, 405-425.

8.Patir, N.; Cheng, H.S., Application of average flow model to lubrication between rough sliding surfaces. J Lubric Tech-T Asme 197\{Craig, 1968 \#4933;Wu, 2016 \#3244\}9, 101, 220.

9.Greenwood, J.A.; Tripp, J.H., The contact of two nominally flat rough surfaces. P I Mech Eng 1970, 185,625 .

10.Bukovnik, S.; Dorr, N.; Caika, V.; Bartz, W.J.; Loibnegger, B., Analysis of diverse simulation models for combustion engine journal bearings and the influence of oil condition. Tribol Int 2006, $39,820-826$.

11.Wei, L.D.; Wei, H.J.; Duan, S.L.; Zhang, Y., An ehd-mixed lubrication analysis of main bearings for diesel engine based on coupling between flexible whole engine block and crankshaft. Ind Lubr Tribol 2015, 67, 150-158.

12.Zhang, Q.-H.; Zheng, X.; Zhang, H.-Y.; Mao, J.; Song, P.; Hao, Z.-Y., An improved tehl model for main bearings of ic engine with flexible crankshaft and block tehl. Journal of the Chinese Society of Mechanical Engineers, Transactions of the Chinese Institute of Engineers, Series C/Chung-Kuo Chi Hsueh Kung Ch'eng Hsuebo Pao 2018, 39, 365-374.

13.Mihara, Y.; Someya, T., Measurement of oil-film pressure in engine bearings using a thin-film sensor. Tribol T 2002, 45, 11-20.

14.Moreau, H.; Maspeyrot, P.; Bonneau, D.; Frene, J., Comparison between experimental film thickness measurements and elastohydrodynamic analysis in a connecting-rod bearing. P I Mech Eng J-J Eng 2002, 216, 195-208.

15.Leclere, Q.; Pezerat, C.; Laulagnet, B.; Polac, L., Indirect measurement of main bearing loads in an operating diesel engine. J Sound Vib 2005, 286, 341-361. 
16.Sun, J.; Zhu, X.L.; Zhang, L.; Wang, X.Y.; Chai, X.H.; Yin, W.; Shi, W., Experimental research on a three-dimensional journal orbit of a crankshaft bearing for an internal combustion engine. J Tribol-T Asme 2014, 136.

17.Allmaier, H.; Priestner, C.; Reich, F.M.; Priebsch, H.H.; Forstner, C.; Novotny-Farkas, F., Predicting friction reliably and accurately in journal bearings - the importance of extensive oilmodels. Tribol Int 2012, 48, 93.

18.Priestner, C.; Allmaier, H.; Priebsch, H.H.; Forstner, C., Refined simulation of friction power loss in crank shaft slider bearings considering wear in the mixed lubrication regime. Tribol Int 2012, 46, 200-207.

19.Ding, N.; Li, H.; Yin, Z.; Zhong, N.; Zhang, L., Journal bearing seizure degradation assessment and remaining useful life prediction based on long short-term memory neural network. Measurement 2020, 166, 108215.

20.Dong, Q.; Yin, Z.; Li, H.; Gao, G.; Zhong, N.; Chen, Y., Simulation and experimental verification of fatigue strength evaluation of journal bearing bush. Eng Fail Anal 2020, 109, 104275.

21.Stachowiak, G.W.; Batchelor, A.W., Engineering tribology. 4 ed.; Butterworth-Heinemann: 2014.

\section{List of Nomenclature}

M Mass matrix of a flexible body

C Damping matrix

K Stiffness matrix

F $\quad$ Force

x Circumferential direction

$\mathrm{z} \quad$ Axial direction

$\theta \quad$ Oil filling factor

$\mathrm{h}, \overline{\mathrm{h}} \quad$ Nominal and average oil film thickness

U Journal circumferential speed

$\eta \quad$ Oil viscosity

$\rho \quad$ Oil density

$\sigma \quad$ Combined surface roughness

$\phi_{x} \quad$ Flow factor in axial direction $\phi_{\mathrm{z}} \quad$ Flow factor in circumferential direction

$\phi_{\mathrm{s}} \quad$ Shear flow factor

$p_{\mathrm{a}} \quad$ Contact pressure created by asperity

contact

K Elastic factor

E' Composite elastic modulus

Hs Dimensionless clearance parameter

$\bar{\delta}_{\mathrm{s}} \quad$ Combined mean summit height

$\mathrm{E}_{1}, \mathrm{E}_{2} \quad$ Young's modulus of the adjacent surfaces

$\varepsilon_{1}, \varepsilon_{2} \quad$ Poisson ratio of the adjacent surfaces

A,B,C Viscosity temperature coefficients

$\mathrm{T} \quad$ Oil film temperature

$\alpha \quad$ Viscosity pressure coefficients 